\title{
Network Coding with Periodic Recomputation for Minimum Energy Multicasting in Mobile Ad-Hoc Networks
}

\author{
Sukwon Kim, Tracey Ho, Michelle Effros \\ Department of Electrical Engineering \\ California Institute of Technology \\ Pasadena, CA, USA 91125 \\ Email: \{sukwon, tho, effros\}@ caltech.edu
}

\begin{abstract}
We consider the problem of minimum-energy multicast using network coding in mobile ad hoc networks (MANETs). The optimal solution can be obtained by solving a linear program every time slot, but it leads to high computational complexity. In this paper, we consider a low-complexity approach, network coding with periodic recomputation, which recomputes an approximate solution at fixed time intervals, and uses this solution during each time interval. As the network topology changes slowly, we derive a theoretical bound on the performance gap between our suboptimal solution and the optimal solution. For complexity analysis, we assume that interior-point method is used to solve a linear program at the first time slot of each interval. Moreover, we can use the suboptimal solution in the preceding interval as a good initial solution of the linear program at each fixed interval. Based on this interior-point method with a warm start strategy, we obtain a bound on complexity. Finally, we consider an example network scenario and minimize the complexity subject to the condition that our solution achieves a given optimality gap.
\end{abstract}

\section{INTRODUCTION}

In this paper, we consider the problem of establishing minimum-energy multicast connections using network coding in mobile ad hoc networks (MANETs). In a static ad hoc network, this problem can be formulated as a linear program for linear and separable cost functions [5], unlike the case without coding which is NP-hard [6]. However, in a mobile scenario, where the locations of nodes in the network change over time, it may still be computationally unattractive to solve the linear optimization at every time slot.

In this paper, we investigate an approximate solution approach based on periodic recomputation. This approach comes from an intuition that when network topology changes slowly, small perturbations occur in the original optimization problem and the original solution remains relatively close to the new optimal solution. In the strategy, time is divided into equal intervals and a suboptimal solution is computed at the first time slot of each interval. As the network topology changes slowly, we use the resulting coding subgraph as a suboptimal solution during each interval. We first derive a bound on the maximum percentage deviation from the optimal cost in terms of the percentage deviation in the cost vector coefficients. For complexity analysis, we assume that barrier and interior-point method is used to solve a linear program at the first time slot of each interval. When we recompute a solution, the linear optimization problem is a slight perturbation of that of the previous time interval as the network topology changes slowly. Then the suboptimal solution in the preceding interval can be used as a warmstart point for interior-point method. By combining our cost bound with this warm start strategy, we obtain a bound on complexity. Finally, we present an example network scenario. In that scenario, we first evaluate our suboptimal strategy and the cost bound. We also consider an optimization problem that minimizes the time complexity subject to the condition that suboptimal solution in the interval achieves a given optimality gap during the interval.

\section{A. Overview}

We continue the introduction with a short review of related work in Section I-B. Section II introduces the system model and formulates the problem. We describe our approach and obtain the theoretical cost bound in Section III. In Section $\mathrm{IV}$, we derive the time-complexity from interior-point method with a warm-start strategy. We present a network scenario in Section V. Section VI concludes this paper.

\section{B. Related Work}

Here we briefly review related works on network optimization in MANETs for the case without coding.

In [10], Zhang et al. investigated energy-efficient packet routing in a multi-hop wireless network with a deterministic mobility model. They considered the objective of minimizing the energy consumption for packet delivery, subject to the packet delay constraint and SINR requirement among concurrent transmissions.

Rodoplu and Meng [11] describe a distributed positionbased network protocol optimized for minimum energy consumption in mobile wireless networks that support peerto-peer communications. They illustrate that a simple local optimization scheme executed at each node guarantees strong connectivity of the entire network and attains the global minimum energy solution for stationary networks.

Above works consider energy-efficient communication problems in MANETs without coding. Each of them formulates the optimization problem and proposes a strategy to solve the problem with specific mobility model. In our paper, we also consider an optimization problem that minimizes the energy consumption in MANETs. We assume the use of network coding and develop a suboptimal strategy with lower complexity. Since we do not assume specific mobility 
model in our strategy, our bounds on the performance gap and complexity can be used as a general bound for any mobility model.

\section{Problem formulation}

In this section, we formulate the minimum-energy multicast problem with network coding in MANETs using linear programming. We adopt the framework from [3] which models a wireless network with a directed hypergraph $\mathcal{H}=$ $(\mathcal{N}, \mathcal{A})$, where $\mathcal{N}$ is the set of nodes and $\mathcal{A}$ is the set of hyperarcs. Each hyperarc $(i, J)$ represents a loseless broadcast link from node $i$ to nodes in nonempty set $J \subset \mathcal{N}$. $z_{i J}$ denotes the rate at which coded packets are injected into hyperarc $(i, J)$. The rate vector $z$ consisting of entries $z_{i J}$ defines a coding subgraph for the multicast connection.A linear and separable cost function $F$ maps valid rate vectors to real numbers. $d_{i, j}$ denotes the Euclidean distance between nodes $i$ and $j$ in the network and we assume for simplicity that $c_{i J}$ $=\max _{j \in J} d_{i, j}$. Then, network cost $F(z)=\sum_{(i, J) \in \mathcal{A}} c_{i J} z_{i J}$. The source node $s$ transmits packets at a positive, real rate $R$ to a nonempty set of sink nodes $T$.

We extend the above formulation to the problem in MANETs by introducing a discrete time dimension. As nodes move, the network topology and link costs change over time. We periodically update the hyperarc set, assuming that any two nodes $i$ and $j$ are connected if $d_{i, j}<D$, where $D$ is a given threshold distance. We use $c_{i J}^{(k)}$ and $\mathcal{A}^{(k)}$ to denote the cost of hyperarc $(i, J)$ and the hyperarc set at the $k$ th time slot, respectively. Then the optimization problem at the $k$ th time slot in MANETs can be formulated as follows:

$$
\begin{array}{cl}
\min & \sum_{\left\{(i, J) \in \mathcal{A}^{(k)}\right\}} c_{i J}^{(k)} z_{i J}^{(k)} \\
& z_{i J}^{(k)} \geqslant \sum_{\{j \in J\}} x_{i J j}^{(t, k)}, \forall(i, J) \in \mathcal{A}^{(k)}, t \in T \\
& \sum_{\left\{J \mid(i, J) \in \mathcal{A}^{(k)}\right\}} \sum_{\{j \in J\}} x_{i J j}^{(t, k)}-\sum_{\left\{j \mid(j, I) \in \mathcal{A}^{(k)}, i \in I\right\}} x_{j I i}^{(t, k)}=\sigma_{i}^{(t)} \\
& \forall i \in \mathcal{N}, t \in T . \\
& x_{i J j}^{(t, k)} \geqslant 0, \forall(i, J) \in \mathcal{A}^{(k)}, j \in J, t \in T
\end{array}
$$

where

$$
\sigma_{i}^{(t)}= \begin{cases}R & \text { if } i=s \\ -R & \text { if } i \in T \\ 0 & \text { otherwise }\end{cases}
$$

We use $L^{(k)}$ to denote this linear program (2) at the $k$ th time slot. By solving $L^{(k)}$, we obtain the global optimal solution $\left(X^{(k)}\right)^{*}=\left\{x_{i J j}^{(t, k)} \mid(i, J) \in \mathcal{A}, j \in J, t \in T\right\}$. Let $C^{(k)}=\left\{c_{i J}^{(k)} \mid(i, J) \in \mathcal{A}\right\}$ and $\left(Z^{(k)}\right)^{*}=\left\{z_{i J}^{(k)} \mid(i, J) \in \mathcal{A}\right\}$ be the network cost vector at time $k$ and the optimal rate vector corresponding to the optimal solution $\left(X^{(k)}\right)^{*}$, respectively. An optimal solution for the problem in MANETs can be obtained by solving (2) every time slot, but it leads to high computational complexity.

\section{NETWORK CODING WITH PERIODIC RECOMPUTATIONS}

Instead of solving (2) every time slot, we propose a suboptimal strategy with lower computational complexity, network coding with periodic recomputation.

\section{A. Algorithm}

$\overline{\text { Algorithm } 1 \text { Algorithm for network coding with periodic }}$ recomputation

if $k \equiv 0 \bmod (p w)$

Reconstruct a hyperarc set $\mathcal{A}^{(k)}$. Given $c_{i J}^{(k)}$ and $\mathcal{A}^{(k)}$, solve $L^{(k)}$ with an optimality gap $\epsilon_{k}$ using interior-

point method and obtain a suboptimal solution $\left(Z^{(k)}, X^{(k)}\right)$.

else if $k \equiv 0 \bmod (p)$

Given $c_{i J}^{(k)}$ and $\mathcal{A}^{(k)}$, solve $L^{(k)}$ with an optimality gap

$\epsilon_{k}$

using interior-point method. We use $\left(Z^{(k-p)}, X^{(k-p)}\right)$

as a feasible warm start point of interior-point method and obtain a suboptimal solution $\left(Z^{(k)}, X^{(k)}\right)$. else

$$
\left(Z^{(k)}, X^{(k)}\right)=\left(Z^{\left(k_{1} \cdot p\right)}, X^{\left(k_{1} \cdot p\right)}\right) \text { where } k_{1}=\left\lfloor\frac{k}{p}\right\rfloor .
$$
end if

In the algorithm, time is divided into intervals where each interval contains $p$ time slots. We recompute an approximate solution in the first time slot of each interval using interior point method and use the resulting coding subgraph as a suboptimal solution during each interval. We assume that the hyperarc set is reconstructed every $w$ intervals, i.e., every $p w$ time slots, and remains the same over $w$ intervals. Reconstructing hyperarc set periodically can cause loss of optimality, but it allows to use the solution computed at the first time slot of interval as a feasible suboptimal solution over interval. When $k \equiv 0 \bmod (p w)$, we recompute the suboptimal solution $\left(Z^{(k)}, X^{(k)}\right)$ by solving $L^{(k)}$ using interior-point method until an optimality gap $\epsilon_{k}$ is achieved, as shown in (2).

$$
\sum_{\{(i, J) \in \mathcal{A}\}} c_{i J}^{k} z_{i J}^{k} \leqslant \sum_{\{(i, J) \in \mathcal{A}\}} c_{i J}^{k}\left(z_{i J}^{k}\right)^{*}+\epsilon_{k} .
$$

In this case, we use any feasible solution as a starting point of interior-point method. When $k \equiv 0 \bmod (p)$ and $k \not \equiv 0 \bmod (p w)$, we solve $L^{(k)}$ with an optimality gap $\epsilon_{k}$ using interior-point method. Here we can use a suboptimal solution $\left(Z^{(k-p)}, X^{(k-p)}\right)$ computed at the first time slot of previous interval as a feasible warm start point of interiorpoint method since the hyperarc set remains the same. When $k \not \equiv 0 \bmod (p)$, we use the suboptimal solution computed at the first time slot of interval which contains the $k$ th time slot as a feasible suboptimal solution. Since by assumption only coefficients of the network cost vector change during each interval, the set of feasible solutions remain the same in the interval. Therefore, we can use the coding subgraph obtained in the first time slot of the interval as a feasible suboptimal solution during each interval. 


\section{B. Theoretical Cost Bound}

In this section, we derive a theoretical bound on the performance gap between our suboptimal solution and the optimal solution. In our algorithm, a suboptimal solution is computed in the first slot of each interval, whose cost deviates from the optimal by at most a given optimality gap. We find a bound on the resulting loss when we use the same solution over the entire interval despite changes in the objective function coefficients.

First, we introduce a useful Lemma by Oguz [7]. This Lemma upper bounds the maximum percentage deviation in the objective function from optimality in terms of the percentage deviation in the objective function coefficients when we stick to the same solution. We consider the following two instances of a general form optimization problem:

$$
\begin{aligned}
& \min z_{1}=\left\{C_{1} X \mid X \in S\right\}, \\
& \min z_{2}=\left\{C_{2} X \mid X \in S\right\} .
\end{aligned}
$$

where $C_{k}=\left(c_{1}^{k}, . ., c_{n}^{k}\right) \in R_{+}^{n}$ is an objective coefficient vector for $k=1,2$ and $S$ is an arbitrary closed and bounded, nonempty set in $R_{+}^{n}$. Let $X_{1}^{*}$ and $X_{2}^{*}$ be the optimal solutions of the above two problems with $z_{1}=C_{1} X_{1}^{*}$ and $z_{2}=C_{2} X_{2}^{*}$, respectively. We assume that $c_{i}^{1}=0$ implies $c_{i}^{2}=0$.

Lemma 1: If

$$
\frac{\left|c_{i}^{1}-c_{i}^{2}\right|}{c_{i}^{1}} \leqslant \epsilon
$$

for all $i$ such that $c_{i}^{1} \neq 0$, then

$$
\frac{z_{2}-z_{3}}{z_{2}} \leqslant \frac{2 \epsilon}{1-\epsilon} \text {. }
$$

where $z_{3}=C^{2} X_{1}^{*}$.

Proof: Please see [7].

We extend the above Lemma 1 to the following result which states that small perturbations in the network cost vector during the interval leave the suboptimal solution computed at the start of the interval, relatively close to the optimal solutions in the interval.

Lemma 2: If $C^{(m p)} Z^{(m p)} \leqslant C^{(m p)}\left(Z^{(m p)}\right)^{*}+\epsilon_{m p}$ and

$$
\begin{gathered}
\max _{(i, J) \in \mathcal{A}} \max _{0 \leqslant j \leqslant p} \frac{\left|c_{i J}^{(m p)}-c_{i J}^{(m p+j)}\right|}{c_{i J}^{(m p)}}=\delta_{m p}, \quad \text { then } \\
C^{(m p+l)} Z^{(m p)} \leqslant \frac{1+\delta_{m p}}{1-\delta_{m p}} C^{(m p+l)}\left(Z^{(m p+l)}\right)^{*}+\left(1+\delta_{m p}\right) \epsilon_{m p},
\end{gathered}
$$

for $\forall 0 \leqslant l \leqslant p$.

Proof: $\quad$ Since $\frac{\left|c_{i J}^{(m p)}-c_{i J}^{(m p+j)}\right|}{c_{i J}^{(m p)}} \leqslant \delta_{m p}$ for $\forall(i, J) \in \mathcal{A}$ and $\forall 0 \leqslant j \leqslant p$ for which $c_{i J}^{(m p)} \neq 0$,

$$
\left(1-\delta_{m p}\right) C^{(m p)} \leqslant C^{(m p+l)} \leqslant\left(1+\delta_{m p}\right) C^{(m p)}, \quad \forall 0 \leqslant l \leqslant p .
$$

By postmultiplying $\left(Z^{(m p+l)}\right)^{*}$ and $Z^{(m p)}$ to the left and right inequalities respectively,

$$
\begin{gathered}
\left(1-\delta_{m p}\right) C^{(m p)}\left(Z^{(m p+l)}\right)^{*} \leqslant C^{(m p+l)}\left(Z^{(m p+l)}\right)^{*}, \\
C^{(m p+l)} Z^{(m p)} \leqslant\left(1+\delta_{m p}\right) C^{(m p)} Z^{(m p)} .
\end{gathered}
$$

Since $C^{(m p)} Z^{(m p)} \leqslant C^{(m p)}\left(Z^{(m p)}\right)^{*}+\epsilon_{m p}$,

$$
\begin{aligned}
& C^{(m p+l)} Z^{(m p)} \\
& \quad \leqslant\left(1+\delta_{m p}\right) C^{(m p)} Z^{(m p)} \\
& \quad \leqslant\left(1+\delta_{m p}\right)\left(C^{(m p)}\left(Z^{(m p)}\right)^{*}+\epsilon_{m p}\right) \\
& \quad \leqslant\left(1+\delta_{m p}\right)\left(C^{(m p)}\left(Z^{(m p+l)}\right)^{*}+\epsilon_{m p}\right) .
\end{aligned}
$$

The last inequality is obtained from the optimality of $\left(Z^{(m p)}\right)^{*}$. Then, by (3),

$C^{(m p+l)} Z^{(m p)} \leqslant \frac{1+\delta_{m p}}{1-\delta_{m p}} C^{(m p+l)}\left(Z^{(m p+l)}\right)^{*}+\left(1+\delta_{m p}\right) \epsilon_{m p}$,

for $\forall 0 \leqslant l \leqslant p$.

This bounds the optimality gap over the interval in terms of the maximum percentage deviation in the cost vector coefficients during the interval and the optimality gap of our suboptimal solution at the first time slot of the interval.

\section{COMPleXity}

When the suboptimal solution is recomputed at the first time slot of each interval, the linear program (2) can be solved using barrier and interior-point method as shown in $[8,9]$. Convergence analysis of barrier and interior-point method for linear optimization problem is given in e.g. [8, Sec. 11.5], where computational complexity is defined in terms of the total number of Newton iterations. In the first time slot of each interval, the linear optimization problem is a slight perturbation of that of the previous time interval as network topology changes slowly. When the hyperarc set is not changed, we can use the suboptimal solution in the preceding interval as the feasible warm-start point for interior point method at the first time slot of following interval. Combining our cost bound with a warm-start strategy using interior point method gives a worst-case bound on the number of Newton iterations required to achieve a given optimality gap. $\left(Z^{(k)}(0), X^{(k)}(0)\right)$ and $q^{(k)}$ are used to denote the feasible starting subgraph for $L^{(k)}$ and the optimal cost of $L^{(k)}$, respectively. From [8-9], an upper bound on the total number of Newton steps for $L^{(k)}, N^{(k)}$, is given as follows:

$$
\begin{aligned}
& N^{(k)}=G\left\lceil\sqrt{M} \log _{2}\left(\frac{\left(C^{(k)} Z^{(k)}(0)-C^{(k)}\left(Z^{(k)}\right)^{*}\right)}{\epsilon_{k}}\right)\right\rceil \\
& \leqslant G\left(\sqrt{M} \log _{2}\left(\frac{C^{(k)} Z^{(k)}(0)-q^{(k)}}{\epsilon_{k}}\right)+1\right)
\end{aligned}
$$

where $M$ is the number of inequalities in $L^{(k)}, \epsilon_{k}$ is the required optimality gap, and $G=11.5$.

In our algorithm, the suboptimal solution $\left(Z^{(m p)}, X^{(m p)}\right)$ is recomputed at time $m p$ for $\forall m \geqslant 0$. Since the hyperarc set is reconstructed every $w$ intervals, the feasible solution set is accordingly changed every $w$ intervals. Thus, if $m \equiv 0(\bmod w)$, we first find any feasible solution of $L^{(m p)}$ and start interior-point method from that point. Otherwise, $\left(Z^{(m-1) p}, X^{(m-1) p}\right)$ is used as the suboptimal solution during the interval $((m-1) p, m p-1)$, and it is also used as a feasible starting point of interior-point method at 
time $m p$, i.e. $\left(Z^{(m p)}(0), X^{(m p)}(0)\right)=\left(Z^{(m-1) p}, X^{(m-1) p}\right)$. Here we define the normalized complexity during the interval $(m p,(m+1) p-1)$ as the total number of Newton iterations at the first time slot of the interval divided by the interval length, i.e. $\frac{N^{(m p)}}{p}$. By combining Lemma 2 with (4), we can obtain an upper bound on the normalized complexity as follows.

Theorem 1: For $m \not \equiv 0 \bmod w$, the normalized complexity over interval $(m p,(m+1) p-1)$ is at most

$$
\frac{G}{p}\left(\sqrt{M} \log _{2}\left(f\left(\delta_{(m-1) p}, \epsilon_{(m-1) p}, \epsilon_{m p}, q^{(m p)}\right)\right)+1\right),
$$

where

$$
\begin{aligned}
& f\left(\delta_{(m-1) p}, \epsilon_{(m-1) p}, \epsilon_{m p}, q^{(m p)}\right) \\
& \quad=\frac{\frac{2 \delta_{(m-1) p}}{1-\delta_{(m-1) p}} q^{(m p)}+\left(1+\delta_{(m-1) p}\right) \epsilon_{(m-1) p}}{\epsilon_{m p}} .
\end{aligned}
$$

Proof: $\quad$ Since $m \quad \not \quad 0(\bmod w)$, $\left(Z^{(m p)}(0), X^{(m p)}(0)\right)=\left(Z^{(m-1) p}, X^{(m-1) p}\right)$. Then, from (4),

$$
\begin{aligned}
& \frac{N^{(m p)}}{p} \\
& \leqslant \frac{G}{p}\left(\sqrt{M} \log _{2}\left(\frac{C^{(m p)} Z^{(m p)}(0)-q^{(m p)}}{\epsilon_{m p}}\right)+1\right) \\
& =\frac{G}{p}\left(\sqrt{M} \log _{2}\left(\frac{C^{(m p)} Z^{(m-1) p}-q^{(m p)}}{\epsilon_{m p}}\right)+1\right) .
\end{aligned}
$$

From Lemma 2,

$$
\begin{aligned}
& C^{(m p)} Z^{(m-1) p}-q^{(m p)} \\
& \leqslant \frac{1+\delta_{(m-1) p}}{1-\delta_{(m-1) p}} q^{(m p)}+\left(1+\delta_{(m-1) p}\right) \epsilon_{(m-1) p}-q^{(m p)} \\
& =\frac{2 \delta_{(m-1) p}}{1-\delta_{(m-1) p}} q^{(m p)}+\left(1+\delta_{(m-1) p}\right) \epsilon_{(m-1) p} \\
& =\epsilon_{m p} f\left(\delta_{(m-1) p}, \epsilon_{(m-1) p}, \epsilon_{m p}, q^{(m p)}\right) .
\end{aligned}
$$

Then,

$$
\begin{aligned}
& \frac{N^{(m p)}}{p} \\
& \leqslant \frac{G}{p}\left(\sqrt{M} \log _{2}\left(f\left(\delta_{(m-1) p}, \epsilon_{(m-1) p}, \epsilon_{m p}, q^{(m p)}\right)\right)+1\right) .
\end{aligned}
$$

Corollary 1: If we require a fractional performance gap $\frac{\epsilon_{m p}}{q^{(m p)}}=\frac{1}{\gamma}$ for $\forall m \geqslant 0$ in the first time slot of each interval, then for each interval $(m p,(m+1) p-1), m \not \equiv 0 \bmod w$, the normalized complexity is at most

$$
\frac{G}{p}\left(\sqrt{M} \log _{2}\left(g\left(\delta_{(m-1) p}, \delta_{m p}, \gamma\right)\right)+1\right),
$$

where

$$
g\left(\delta_{(m-1) p}, \gamma\right)=\frac{2 \delta_{(m-1) p} \gamma+\left(1+\delta_{(m-1) p}\right)}{1-\delta_{(m-1) p}} .
$$

Proof: From Theorem 1,

$$
\begin{aligned}
f & \left(\delta_{(m-1) p}, \epsilon_{(m-1) p}, \epsilon_{m p}, q^{(m p)}\right) \\
& =\frac{2 \delta_{(m-1) p} \gamma}{1-\delta_{(m-1) p}}+\frac{\left(1+\delta_{(m-1) p}\right) \epsilon_{(m-1) p}}{\epsilon_{m p}} .
\end{aligned}
$$

By (3) in the proof of Lemma 2,

$$
\left(1-\delta_{(m-1) p}\right) C^{(m-1) p}\left(Z^{(m p)}\right)^{*} \leqslant C^{(m p)}\left(Z^{(m p)}\right)^{*} .
$$

From the optimality of $\left(Z^{(m-1) p}\right)^{*}$, we obtain

$$
\begin{aligned}
(1 & \left.-\delta_{(m-1) p}\right) q^{(m-1) p} \\
& =\left(1-\delta_{(m-1) p}\right) C^{(m-1) p}\left(Z^{(m-1) p}\right)^{*} \\
& \leqslant\left(1-\delta_{(m-1) p}\right) C^{(m-1) p}\left(Z^{(m p)}\right)^{*} \\
& \leqslant C^{(m p)}\left(Z^{(m p)}\right)^{*}=q^{(m p)} .
\end{aligned}
$$

Then,

$$
\frac{q^{(m-1) p}}{q^{(m p)}}=\frac{\epsilon_{(m-1) p}}{\epsilon_{m p}} \leqslant \frac{1}{1-\delta_{(m-1) p}}
$$

and thus,

$$
\begin{aligned}
& f\left(\delta_{(m-1) p}, \epsilon_{(m-1) p}, \epsilon_{m p}, q^{(m p)}\right) \\
& \quad \leqslant \frac{2 \delta_{(m-1) p} \gamma+\left(1+\delta_{(m-1) p}\right)}{1-\delta_{(m-1) p}} \\
& \quad=g\left(\delta_{(m-1) p}, \gamma\right) .
\end{aligned}
$$

Therefore,

$$
\begin{aligned}
& \frac{N^{(m p)}}{p} \\
& \leqslant \frac{G}{p}\left(\sqrt{M} \log _{2}\left(f\left(\delta_{(m-1) p}, \epsilon_{(m-1) p}, \epsilon_{m p}, q^{(m p)}\right)\right)+1\right) \\
& \leqslant \frac{G}{p}\left(\sqrt{M} \log _{2}\left(g\left(\delta_{(m-1) p}, \gamma\right)\right)+1\right) .
\end{aligned}
$$

Corollary 1 gives an upper bound on the normalized complexity that grows logarithmically with $\gamma$. This result matches the intuition that the amount of computation increases as higher precision is required.

\section{Evaluation}

In this section, we present an example network scenario for which we evaluate our suboptimal strategy and the cost bound in the scenario. We also optimize the normalized complexity subject to the condition that suboptimal solution in the interval achieves a given optimality gap during the interval. 


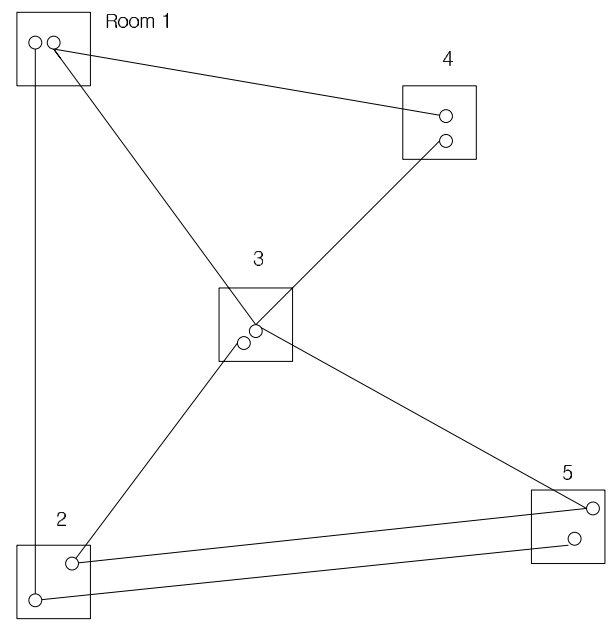

Fig. 1. Scenario: 5 rooms are distributed over large area. Distance between any two rooms are sufficiently large. Each room contains two nodes and each node is connected to at least one node in different room.

\section{A. Scenario}

We consider a scenario in which $m$ square rooms are distributed over same area, as shown in Fig. 1. Each room contains several nodes, total $n$ nodes. Hypergraph $\mathcal{H}=$ $(\mathcal{N}, \mathcal{A})$ is defined on this network. To communicate with nodes in different rooms, for any hyperarc $(i, J)$, we assume that the set of destination nodes $J$ contains at least one node contained in a different room from $i . d_{0}$ is used to denote the minimum distance between any two rooms. Then, by the definition of hyperarc cost, $c_{i J} \geqslant d_{0}$ for $\forall(i, J) \in \mathcal{A}$. Here we use a mobility model based on a two-dimensional random walk model. The initial location of each node is given, and each node in each room moves as a random walker on a two-dimensional lattice. Each node has a probability of $\frac{1}{4}$ of moving to a position above, below, to the left, or to the right of its current position with step size $\alpha$ every time slot. When a node reaches a boundary of the room, it is reflected.

\section{B. Cost Bound}

Here we evaluate our suboptimal strategy and the cost bound for above scenario. From Lemma 2, the cost bound increases with the maximum percentage deviation in the objective function coefficients during an interval. We will show that the cost bound for above scenario can be expressed in terms of $p, d_{0}$, and $\alpha$. It is also shown that the cost bound grows as the length of interval and the speed of nodes. Before proving that, we introduce an useful Lemma.

Lemma 3: Given any two finite sequences $\left\{a_{0}, a_{1}, ..\right\}$ and $\left\{b_{0}, b_{1}, ..\right\}$

$$
\max _{i}\left|a_{i}-b_{i}\right| \geqslant\left|\max _{k} a_{k}-\max _{t} b_{t}\right| .
$$

Proof: Suppose that $\max _{k}^{i} a_{k}=a_{l}$ and $\max _{t} b_{t}=b_{s}$.

Case 1): $a_{l} \geqslant b_{s}$.

Since $b_{s} \geqslant b_{l},\left|\max _{k} a_{k}-\max _{t} b_{t}\right|=\left|a_{l}-b_{s}\right|=a_{l}-b_{s} \leqslant$ $a_{l}-b_{l}=\left|a_{l}-b_{l}\right| \leqslant \max _{i}\left|a_{i}-b_{i}\right|$.

Case 2): $a_{l}<b_{s}$.

Since $a_{l} \geqslant a_{s},\left|\max _{k} a_{k}-\max _{t} b_{t}\right|=\left|a_{l}-b_{s}\right|=b_{s}-a_{l} \leqslant$ $b_{s}-a_{s}=\left|b_{s}-a_{s}\right| \leqslant \max _{i}\left|b_{i}-a_{i}\right|=\max _{i}\left|a_{i}-b_{i}\right|$.
Using Lemma 3, we prove Theorem 2 which states that the cost bound during an interval grows with the length of interval and the step size of random walk.

Theorem 2: For $\forall 0 \leqslant l \leqslant p$ and $m \geqslant 0$,

$$
\begin{aligned}
C^{(m p+l)} Z^{(m p)} & \leqslant\left(\frac{1+\frac{2 \alpha p}{d_{0}}}{1-\frac{2 \alpha p}{d_{0}}}\right) C^{(m p+l)}\left(Z^{(m p+l)}\right)^{*} \\
& +\left(1+\frac{2 \alpha p}{d_{0}}\right) \epsilon_{m p} .
\end{aligned}
$$

Proof: From Theorem 1, it is enough to show $\delta_{m p} \leqslant$ $\frac{2 \alpha p}{d_{0}}$ for $m \geqslant 0$. We use $d_{i, j}^{(k)}$ to denote the distance between nodes $i$ and $j$ at time $k$.

$\delta_{m p}$

$$
\begin{aligned}
& =\max _{(i, J) \in \mathcal{A}} \max _{0 \leqslant s \leqslant p} \frac{\left|c_{i J}^{(m p)}-c_{i J}^{(m p+s)}\right|}{c_{i J}^{(m p)}} \\
& \leqslant \max _{(i, J) \in \mathcal{A}} \max _{0 \leqslant s \leqslant p} \sum_{q=1}^{s} \frac{\left|c_{i J}^{(m p+q-1)}-c_{i J}^{(m p+q)}\right|}{c_{i J}^{(m p)}}
\end{aligned}
$$$$
\text { (since }|a+b| \leqslant|a|+|b| \text {.) }
$$

$$
\begin{aligned}
& =\max _{(i, J) \in \mathcal{A}} \sum_{q=1}^{p} \frac{\left|c_{i J}^{(m p+q-1)}-c_{i J}^{(m p+q)}\right|}{c_{i J}^{(m p)}} \\
& =\max _{(i, J) \in \mathcal{A}} \sum_{q=1}^{p} \frac{\left|\max _{j \in J} d_{i, j}^{(m p+q-1)}-\max _{j \in J} d_{i, j}^{(m p+q)}\right|}{\max _{j \in J} d_{i, j}^{(m p)}}
\end{aligned}
$$

(since $c_{i J}^{(k)}=\max _{j \in J} d_{i, j}^{(k)} \cdot$ )

$$
\leqslant \max _{(i, J) \in \mathcal{A}} \sum_{q=1}^{p} \frac{\max _{j \in J}\left|d_{i, j}^{(m p+q-1)}-d_{i, j}^{(m p+q)}\right|}{\max _{j \in J} d_{i, j}^{(m p)}} .
$$

The last inequality is obtained from Lemma 3. From our mobility model, $\left|d_{i, j}^{(k)}-d_{i, j}^{(k+1)}\right| \leqslant 2 \alpha$ for $\forall k \geqslant 0$. Since we assumed that $c_{i J} \geqslant d_{0}$ for $\forall(i, J), \max _{j \in J} d_{i, j}^{(m p)} \geqslant d_{0}$. Therefore,

$$
\max _{\forall(i, J) \in \mathcal{A}} \sum_{q=1}^{p} \frac{\max _{j \in J}\left|d_{i, j}^{(m p+q-1)}-d_{i, j}^{(m p+q)}\right|}{\max _{j \in J} d_{i, j}^{(m p)}} \leqslant \frac{2 \alpha p}{d_{0}} .
$$

Therefore, the cost bound grows with $\alpha$ and $p$. This result matches the intuition that as the speed of nodes increases or as the length of an interval increases, our suboptimal solution may deviate further from the optimal solution during an interval.

From simulation, we test our suboptimal strategy and the cost bound in the given scenario. Two square rooms are placed over a network and the distance between two rooms, $d_{0}$, is $10^{4}$. Each room contains 8 nodes. Room 1 contains a source node and room 1 and 2 contain 2 sink nodes, respectively. Suppose that a size of room is 20 , the length of each interval $p=15$, and the step size $\alpha=10$. As shown in Fig. 2, the cost of a suboptimal solution obtained from our strategy is close to optimal cost during an interval. Moreover, since $d_{0}>\alpha$ and $p$ is not too large, our cost bound is also close to the cost of suboptimal solution. 


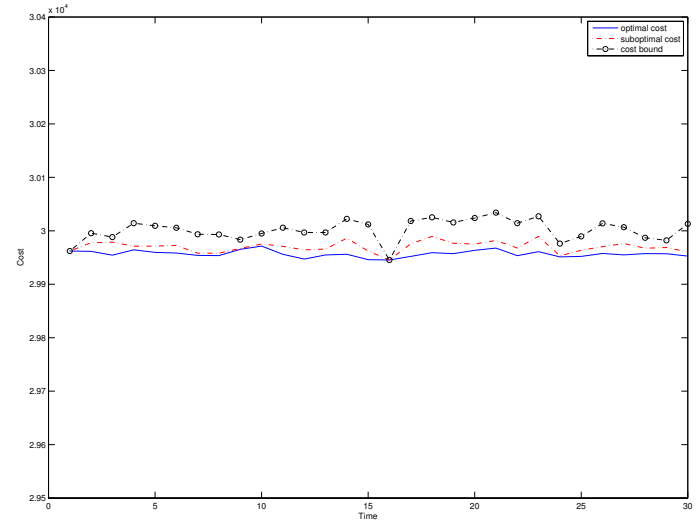

Fig. 2. Simulation result: two rooms are placed on the network and each room contains 8 nodes. $d_{0}=10^{4}, p=15$, and $\alpha=10$. In this scenario, the cost of our suboptimal solution is close to optimal cost.

\section{Complexity}

In this section, we consider an optimization problem that minimizes the normalized complexity subject to the condition that suboptimal solutions in the interval achieve a given optimality gap during the interval. From Theorem 1, we first derive the following upper bound on the normalized complexity can be expressed in terms of interval length $p$ and the fractional optimality gap at the first time slot of each interval, $\frac{1}{\gamma}$.

Corollary 2: For $m \not \equiv 0 \bmod w$, the normalized complexity over interval $(m p,(m+1) p-1)$ is at most

$$
T=\frac{G}{p}\left(\sqrt{M} \log _{2}\left(\frac{\frac{4 \alpha p}{d_{0}} \gamma+\left(1+\frac{2 \alpha p}{d_{0}}\right)}{1-\frac{2 \alpha p}{d_{0}}}\right)+1\right) .
$$

Proof: From (6) in the proof of Theorem 1,

$$
\begin{aligned}
& C^{(m p)} Z^{(m-1) p}-q^{(m p)} \\
& \quad \leqslant \frac{2 \delta_{(m-1) p}}{1-\delta_{(m-1) p}} q^{(m p)}+\left(1+\delta_{(m-1) p}\right) \epsilon_{(m-1) p} .
\end{aligned}
$$

Since it is shown that $\delta_{(m-1) p} \leqslant \frac{2 \alpha p}{d_{0}}$ in the proof of Theorem 2,

$$
\begin{aligned}
& C^{(m p)} Z^{(m-1) p}-q^{(m p)} \\
& \quad \leqslant \frac{\frac{4 \alpha p}{d_{0}}}{1-\frac{2 \alpha p}{d_{0}}} q^{(m p)}+\left(1+\frac{2 \alpha p}{d_{0}}\right) \epsilon_{(m-1) p}
\end{aligned}
$$

Then, from (5) in the proof of Theorem 1,

$$
\begin{aligned}
& \frac{N^{(m p)}}{p} \\
& \leqslant \frac{G}{p}\left(\sqrt{M} \log _{2}\left(\frac{C^{(m p)} Z^{(m-1) p}-q^{(m p)}}{\epsilon_{m p}}\right)+1\right)
\end{aligned}
$$$$
\leqslant \frac{G}{p}\left[\sqrt{M} \log _{2}\left(\frac{\frac{\frac{4 \alpha p}{d_{0}}}{1-\frac{2 \alpha p}{d_{0}}} q^{(m p)}+\left(1+\frac{2 \alpha p}{d_{0}}\right) \epsilon_{(m-1) p}}{\epsilon_{m p}}\right)+1\right]^{\frac{\epsilon_{m p}^{q^{(m p)}} \cdot \frac{1}{\gamma}}{\text { Tast inequality comes from the optimality of }\left(Z^{(m p)}\right)}} \frac{\epsilon_{m p}}{q^{(m p+l)}}=\frac{q^{m p}}{q^{(m p+l)}} \cdot \frac{\epsilon_{m p}}{q^{(m p)}} \leqslant \frac{1}{\left(1-\frac{2 \alpha p}{d_{0}}\right) \gamma} .
$$

Since $\frac{\epsilon_{(m-1) p}}{\epsilon_{m p}} \leqslant \frac{1}{1-\delta_{(m-1) p}}$ from (7) in the proof of Corollary 1 and $\delta_{(m-1) p} \leqslant \frac{2 \alpha p}{d_{0}}$,

$$
\frac{\frac{\frac{4 \alpha p}{d_{0}}}{1-\frac{2 \alpha p}{d_{0}}} q^{(m p)}+\left(1+\frac{2 \alpha p}{d_{0}}\right) \epsilon_{(m-1) p}}{\epsilon_{m p}} \leqslant \frac{\frac{4 \alpha p}{d_{0}} \gamma+\left(1+\frac{2 \alpha p}{d_{0}}\right)}{1-\frac{2 \alpha p}{d_{0}}} .
$$

Therefore,

$$
\frac{N^{(m p)}}{p} \leqslant \frac{G}{p}\left(\sqrt{M} \log _{2}\left(\frac{\frac{4 \alpha p}{d_{0}} \gamma+\left(1+\frac{2 \alpha p}{d_{0}}\right)}{1-\frac{2 \alpha p}{d_{0}}}\right)+1\right)=T \text {. }
$$

Next we show that $T$ grows logarithmically with the maximum percentage deviation in the cost bound of our suboptimal solution. We use $y$ to denote an upper bound on the fractional optimality gap over each interval. (Note that $\left.\frac{1}{\gamma} \leqslant y\right)$.

Lemma 4: Let

$$
y=\frac{1}{1-\frac{2 \alpha p}{d_{0}}}\left(\frac{4 \alpha p}{d_{0}}+\frac{1}{\gamma}\left(1+\frac{2 \alpha p}{d_{0}}\right)\right) .
$$

Then, for $m \not \equiv 0 \bmod w$,

$$
\begin{gathered}
T=\frac{G}{p}\left(\sqrt{M} \log _{2}(y \gamma)+1\right), \quad \text { and } \\
C^{(m p+l)} Z^{(m p)} \leqslant(1+y) q^{(m p+l)} \text { for } \quad \forall 0 \leqslant l \leqslant p .
\end{gathered}
$$

Proof: From Corollary 2, it is obvious that

$$
T=\frac{G}{p}\left(\sqrt{M} \log _{2}(y \gamma)+1\right) .
$$

It is enough to show that $C^{(m p+l)} Z^{(m p)} \leqslant(1+y) q^{(m p+l)}$ for $\forall 0 \leqslant l \leqslant p$. From Theorem 2 ,

$$
C^{(m p+l)} Z^{(m p)} \leqslant \frac{1+\frac{2 \alpha p}{d_{0}}}{1-\frac{2 \alpha p}{d_{0}}} q^{(m p+l)}+\left(1+\frac{2 \alpha p}{d_{0}}\right) \epsilon_{m p} .
$$

Then,

$$
\frac{C^{(m p+l)} Z^{(m p)}}{q^{(m p+l)}} \leqslant \frac{1+\frac{2 \alpha p}{d_{0}}}{1-\frac{2 \alpha p}{d_{0}}}+\frac{\left(1+\frac{2 \alpha p}{d_{0}}\right) \epsilon_{m p}}{q^{(m p+l)}} .
$$

Now we can obtain the following inequalities.

$$
\begin{aligned}
q^{(m p+l)} & =C^{(m p+l)}\left(Z^{(m p+l)}\right)^{*} \\
& \geqslant\left(1-\delta_{m p}\right) C^{(m p)}\left(Z^{(m p+l)}\right)^{*} \\
& \geqslant\left(1-\frac{2 \alpha p}{d_{0}}\right) C^{(m p)}\left(Z^{(m p+l)}\right)^{*} \\
& \geqslant\left(1-\frac{2 \alpha p}{d_{0}}\right) C^{(m p)}\left(Z^{(m p)}\right)^{*} \\
& =\left(1-\frac{2 \alpha p}{d_{0}}\right) q^{(m p)} .
\end{aligned}
$$

First inequality comes from (3) in the proof of Theorem 1 and the second inequality is true since $\delta_{m p} \leqslant \frac{2 \alpha p}{d_{0}}$. The last inequality comes from the optimality of $\left(Z^{(m p)}\right)^{*}$. Since $1 \frac{\epsilon_{m p}}{q^{(m p)}}=\frac{1}{\gamma}$ 
Therefore,

$$
\begin{aligned}
\frac{C^{(m p+l)} Z^{(m p)}}{q^{(m p+l)}} & \leqslant \frac{1+\frac{2 \alpha p}{d_{0}}}{1-\frac{2 \alpha p}{d_{0}}}+\frac{\left(1+\frac{2 \alpha p}{d_{0}}\right)}{\left(1-\frac{2 \alpha p}{d_{0}}\right) \gamma} \\
& =1+y .
\end{aligned}
$$

Based on Lemma 4, we can formulate an optimization problem that minimizes the normalized complexity over $\gamma$ and $p$ subject to the condition that suboptimal solutions in the interval achieve a fractional optimality gap $y$ over each interval. For $m \not \equiv 0 \bmod w$,

$$
\begin{aligned}
\min & \frac{G}{p}\left(\sqrt{M} \log _{2}(y \gamma)+1\right) \\
\text { s.t } & \frac{1}{1-\frac{2 \alpha p}{d_{0}}}\left(\frac{4 \alpha p}{d_{0}}+\frac{1}{\gamma}\left(1+\frac{2 \alpha p}{d_{0}}\right)\right)=y \\
& p, \gamma \geqslant 0 .
\end{aligned}
$$

Assume we are given $d_{0}$ and $\alpha$ such that $\frac{2 \alpha}{d_{0}}=s<<1$. Then (8) is equivalent to following problem:

$$
\begin{aligned}
\min & \frac{G}{p}\left(\sqrt{M} \log _{2}(y \gamma)+1\right) \\
\text { s.t } & \frac{1}{1-s p}\left(2 s p+\frac{1}{\gamma}(1+s p)\right)=y \\
& p, \gamma \geqslant 0 .
\end{aligned}
$$

By using the constraint in (9), $p$ can be expressed in terms of $\gamma$. Then, we can reformulate this problem as follows.

$$
\begin{array}{ll}
\min & f(\gamma)=G s \frac{(1+\gamma(2+y))}{\gamma y-1}\left(\sqrt{M} \log _{2}(y \gamma)+1\right) \\
\text { s.t } \quad \gamma=\frac{1+p s}{y-(2+y) s p} \geqslant \frac{1}{y}
\end{array}
$$

Therefore, by solving (10) for $\gamma \geqslant \frac{1}{y}$, we can obtain the optimal $\gamma^{*}$ and

$$
p^{*}=\frac{\gamma^{*} y-1}{s\left(1+\gamma^{*}(2+y)\right)} .
$$

We prove two propositions that are useful to find the optimal $\left(\gamma^{*}, p^{*}\right)$.

Proposition 1: $f(\gamma)$ has only one global minimum for $\gamma \geqslant \frac{1}{y}$.

Proof: From (10), it is enough to show that

$$
g(\gamma)=\left(1+\frac{2(\gamma+1)}{\gamma y-1}\right)\left(\log (y \gamma)+\frac{1}{\sqrt{M} \log _{2} e}\right) .
$$

has only one global minimum for $\gamma \geqslant \frac{1}{y}$.

$$
\begin{aligned}
g^{\prime}(\gamma)= & \frac{\gamma^{2} y(y+2)-2 \gamma-1}{\gamma(\gamma y-1)^{2}} \\
& -\frac{2 \gamma(y+1)\left(\log (y \gamma)+\frac{1}{\sqrt{M} \log _{2} e}\right)}{\gamma(\gamma y-1)^{2}} \\
= & \frac{h(\gamma)}{\gamma(\gamma y-1)^{2}} .
\end{aligned}
$$

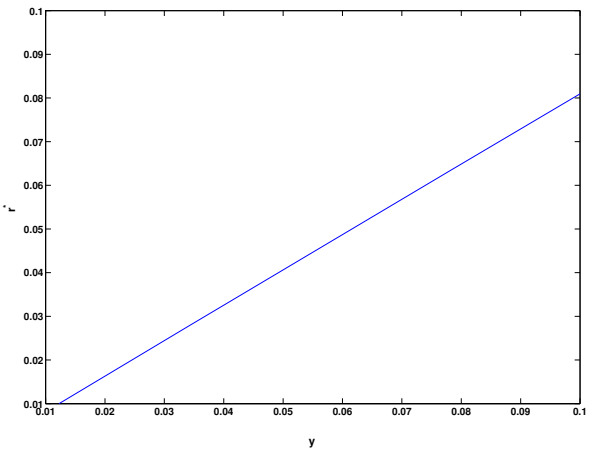

Fig. 3. Optimal fractional performance gap at the start of interval, $1 / \gamma^{*}$ versus $y$, an upper bound on the fractional optimality gap over each interval, when $s=10^{-3}$ and $M=1000$ in (10).

Then, it is enough to show that $h(\gamma)$ has only one root for $\gamma \geqslant \frac{1}{y}$.

$$
\begin{aligned}
& h^{\prime}(\gamma)= 2 \gamma y(y+2)-2 \\
&-2(y+1)\left(\log (y \gamma)+\frac{1}{\sqrt{M} \log _{2} e}+1\right), \\
& h^{\prime \prime}(\gamma)=2 y(y+2)-\frac{2(y+1)}{\gamma} \geqslant 0 \text { for } \gamma>\frac{1}{y} .
\end{aligned}
$$

Then $h^{\prime}(\gamma)$ increases for $\gamma>\frac{1}{y}$. Since $h^{\prime}\left(\frac{1}{y}\right)<0, h^{\prime}$ has only one root $\gamma_{1}$. Therefore, $h$ decreases for $\frac{1}{y}<\gamma \leqslant \gamma_{1}$ and increases for $\gamma>\gamma_{1}$. Since $h\left(\frac{1}{y}\right)=-2(\gamma+1) \frac{1}{\sqrt{M} \log _{2} e}<0$, $h$ has only one root for $\gamma \geqslant \frac{1}{y}$.

Proposition 2: For $500 \leqslant M \leqslant 2500, \frac{1.15}{y}<\gamma^{*}<\frac{1.78}{y}$.

Proof: Let $\gamma y=K$. From the proof of Proposition 1,

$h(\gamma)=K^{2}+2 K \gamma-2 \gamma-1-2(\gamma+K)\left(\log (K)+\frac{1}{\sqrt{M} \log _{2} e}\right)$.

Then,

$$
\begin{aligned}
& K^{2}+2 K \gamma-2 \gamma-1-2(\gamma+K)(\log (K)+0.031 \\
& \quad \leqslant h(\gamma) \\
& \quad \leqslant K^{2}+2 K \gamma-2 \gamma-1-2(\gamma+K)(\log (K)+0.014) .
\end{aligned}
$$

From above inequalities, we obtain

$$
\begin{gathered}
h\left(\frac{1.15}{\gamma}\right) \leqslant-0.07 \gamma-0.2<0, . \\
h\left(\frac{1.78}{\gamma}\right) \geqslant 0.35 \gamma+0.01>0 .
\end{gathered}
$$

Since $h$ has only one root, $\frac{1.15}{y}<\gamma^{*}<\frac{1.78}{y}$ for $500 \leqslant M \leqslant$ 2500.

As shown in Fig. 3, the optimal fractional performance gap at the start of interval, $1 / \gamma^{*}$, grows almost linearly with the upper bound of fractional optimality gap during interval, $y$. Given $\gamma^{*}, p^{*}$ is given by (11). In Fig. 4, the optimal length of interval, $p^{*}$, also grows almost linearly with $y$. Thus, when we allow a larger optimality gap, we recompute the solution less frequently and with less precison. As shown in Fig. 5, 


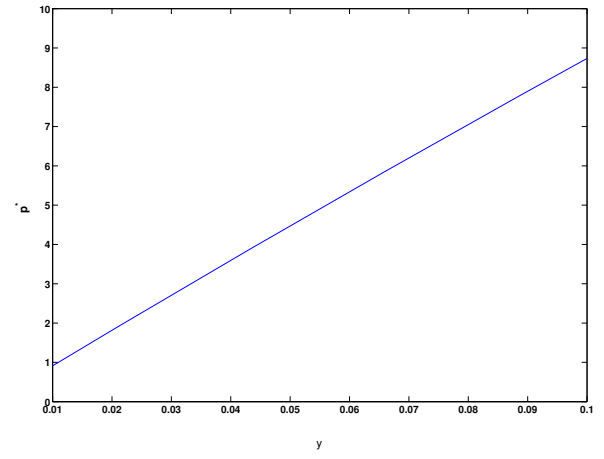

Fig. 4. Optimal length of interval, $p^{*}$, versus $y$ when $s=10^{-3}$ and $M=1000$ in (10).

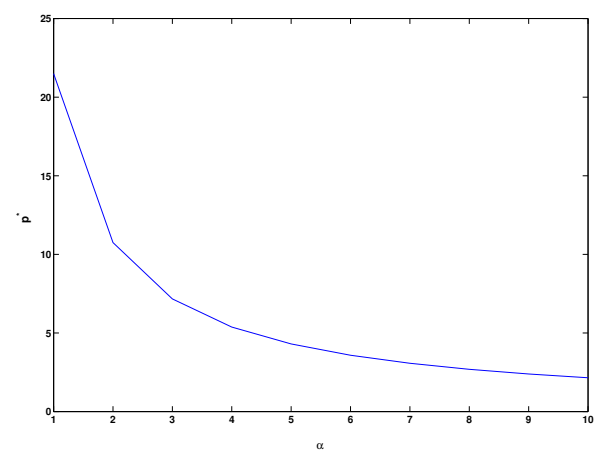

Fig. 5. $p^{*}$ versus the speed of each node, $\alpha$ when $y=0.05$ and $d_{0}=10^{4}$.

when $y$ is fixed, $p^{*}$ decreases as the speed of each node increases. Since the network topology changes faster as $\alpha$ increases, we have to recompute the solution more frequently.

\section{Conclusion And Future Work}

In this paper, we analyze network coding with periodic recomputation for minimum energy multicasting in MANETs. In this approach, we recompute an approximate solution at fixed time intervals, and use this solution during each time interval although the network topology changes. We obtain a simple theoretical cost bound on the gap between our solution and the optimal cost. We also analyze its complexity, and show how our results can be applied to trade off performance and complexity in a given network scenario.

We can also apply our periodic recomputation approach to other network optimization problems in MANETs to reduce the computational complexity. If the set of feasible solutions does not change every time slot, we can directly use our periodic recomputation approach. It is interesting further work to extend our results to the case where this does not hold, and to analyze the performance-complexity tradeoff of other algorithms.

\section{ACKNOWLEDGEMENTS}

This work has been supported in part by the Defense Advanced Research Projects Agency (DARPA) under Contract
No. W911NF-07-1-0029, and by Caltech's Lee Center for Advanced Networking.

\section{REFERENCES}

[1] R. Ahlswede, N. Cai, S.-Y.R.Li, and R. W. Yeung, "Network information flow," IEEE Trans. Inform. Theory, vol 46, no. 4, pp. 1204-1216, July 2000.

[2] M. Efrros, T. Ho, and S. Kim, "A Tiling Approach to Network Code Design for Wireless Networks," in Proc. of Information Theory Workshop(ITW), Punta del Este, Uruguay, Mar. 2006, pp. 62-66.

[3] Y.Wu, P.A. Chou, and S.-Y. Kung. "Minimun-energy multicast in mobile ad-hoc networks using network coding "IEEE Trans. Commun., vol 53, no. 11, pp. 1906-1918, Nov. 2005.

[4] Y.Wu, P.A. Chou, and S.-Y. Kung. "Information exchange in wireless networks with network coding and physical layer broadcast," Technical Report MSR-TR-2004-78, Microsoft Research, Redmond, WA, Aug. 2004.

[5] D. S. Lun, N. Ratnakar, M. Medard, R. Koetter, D. R. Karger, T. Ho, and E. Ahmed, "Minimum-Cost Multicast over Coded Packet Network", IEEE Trans. Inform. Theory, 52(6):2608-2623, June 2006.

[6] J. E. Wieselthier, G. D. Nguyen, and A. Ephremides, "On construction of energy-efficient broadcast and multicast trees in wireless networks". In Proc. IEEE Conf. on Computer Comm. (INFOCOM), pp. 586-594, 2000.

[7] O. Oguz, "Bounds on the opportunity cost of neglecting reoptimization in mathematical programming". In Management Sci. 46(7), pp. 10091012, 2000.

[8] S. Boyd and L. Vandenberghe, Convex optimization. Cambridge, UK ; New York : Cambridge, 2004.

[9] D. P. Bertsekas and J. N. Tsitsiklis, Introduction to linear optimization. Belmont, MA: Athena Scientific, 1997.

[10] J. Zhang, Q. Zhang, B. Li, X. Luo, and W. Zhu, "Energy-efficient routing in mobile ad hoc networks: mobility-assisted case," IEEE Trans. Vehicular Technology, vol 55, no. 1, Jan. 2006, pp. 369-379.

[11] V. Rodoplu and T.H. Meng, "Minimum energy mobile wireless networks," IEEE J. Select. Areas Commun, vol 17, no. 8, Aug. 1999, pp. 1333-1344.

[12] I. T. Haque, and C. Assi, "Randomized energy aware routing algorithms in mobile ad hoc networks," Proc. of the 8th ACM international symposium on Modeling, analysis and simulation of wireless and mobile systems, Montreal, Quebec, Canada, 2005, pp. 71-78.

[13] I. T. Haque, C. Assi, J. W. Atwood, "Localized energy efficient routing in mobile ad hoc networks," Wireless Communications and Mobile Computing, vol 7, no. 6, Aug. 2007, pp. 781-793. 